\title{
Influence of crystal orientation on the thermomechanical fatigue behaviour in a single- crystal superalloy
}

\author{
Mikael Segersäll, Daniel Leidermark and Johan Moverare
}

\section{Linköping University Post Print}

\section{Tweet}

N.B.: When citing this work, cite the original article.

Original Publication:

Mikael Segersäll, Daniel Leidermark and Johan Moverare, Influence of crystal orientation on the thermomechanical fatigue behaviour in a single-crystal superalloy, 2014, Materials Science \&amp; Engineering: A, 623(19), 68-77.

http://dx.doi.org/10.1016/j.msea.2014.11.026

Copyright: Elsevier

http://www.elsevier.com/

Postprint available at: Linköping University Electronic Press

http://urn.kb.se/resolve?urn=urn:nbn:se:liu:diva-111639 


\title{
Influence of crystal orientation on the thermomechanical fatigue behaviour in a single-crystal superalloy
}

\author{
Mikael Segersäll ${ }^{*, a}$, Daniel Leidermark ${ }^{\mathrm{b}}$, Johan J. Moverare ${ }^{\mathrm{a}}$ \\ ${ }^{a}$ Division of Engineering Materials, Linköping University, SE-58183 Linköping, Sweden \\ ${ }^{b}$ Division of Solid Mechanics, Linköping University, SE-58183 Linköping, Sweden
}

\begin{abstract}
In this study, the influence of crystal orientation on the thermomechanical fatigue (TMF) behaviour of the recently developed single-crystal superalloy STAL-15 is considered, both from an experimental and a modelling perspective. Experimental results show that there is a strong influence of the elastic stiffness, with respect to the loading direction, on the TMF life. However, the results also indicate that the number of active slip planes during deformation influence the TMF life, where specimens with a higher number of active slip planes are favoured compared to specimens with fewer active slip planes. The higher number of active slip planes results in a more widespread deformation compared to a more concentrated deformation when fewer slip planes are active. Deformation bands with smeared and elongated $\gamma^{\prime}$-precipitates together with deformation twinning were found to be major deformation mechanisms, where the twins primarily were observed in specimens with several active slip planes. From a modelling perspective, the crystal orientation with respect to
\end{abstract}

\footnotetext{
${ }^{*}$ Corresponding author. Tel: $+46(0) 13281145$

Email address: mikael.segersall@liu.se (Mikael Segersäll)
} 
the loading direction is quantified and adopted into a framework which makes it possible to describe the internal crystallographic arrangement and its entities in a material model. Further, a material model which incorporates the crystal orientation is able to predict the number of slip planes observed from microstructural observations, as well as the elastic stiffness of the material with respect to the loading direction.

Key words: Single-crystal superalloy, Crystal orientation dependence, Thermomechanical fatigue, Deformation mechanisms, Finite element applicability

\section{Introduction}

The need for understanding thermomechanical fatigue (TMF) behaviour of single-crystal nickel-based superalloys is growing for the gas turbine industry. One way to increase the turbine efficiency is to increase the turbine entry temperature, and over a long period of time a lot of effort has been put into developing materials that can withstand higher temperatures, see e.g. [1]. However for the new generation of gas turbines it is not only the turbine entry temperature that needs to be increased, the turbine engine will also operate under different load conditions compared to what has been done previously. Thus, more gas turbines have to be used as a complement for the increasing number of renewable energy sources, for example when the wind is not blowing or when the sun is not shining [2]. This means a higher number of starts and stops of the engine compared to earlier conditions when the engine instead run at steady-state condition over longer periods of time. As the temperature within the turbine is increasing, new and advanced cooling techniques are implemented in the design of hot section components, such as the gas turbine blade. These new cooling techniques result in higher stresses and temperature gradients within the components. More complex stress gradients together with an increasing number of start-ups and 
shut-downs means that TMF testing, where both mechanical strain and temperature are cycled, must be considered in manifesting realistic loading conditions. In addition to TMF testing, the ability to model the TMF behaviour becomes more important for the gas turbine industry when estimating the service lives of hot section components. That the importance of understanding the TMF mechanisms is growing is visible in the literature, where the area of TMF in single-crystal superalloys is expanding $[3,4,5,6,7,8,9,10]$.

Gas turbine components made of single-crystal nickel-base superalloy material are produced by investment casting, see e.g. [11]. The preferred growth direction for nickel, and other face-centered-cubic (FCC) alloys, is the [001] crystal direction [12]. This direction is also beneficial from a fatigue point of view, since this direction has the lowest stiffness and therefore the best fatigue properties under strain-controlled fatigue. In the casting process a grain selector is used to single-out a grain which is allowed to expand in the mould. This grain selector has a large influence on the crystallographic orientation of the component, and it has been shown that the length and angle of the spiral of the grain selector are critical parameters [13]. To further improve the crystal orientation of the components in the casting process, a cellular automation finite element (FE) simulation can be performed to investigate the grain density and texture prior casting $[14,15]$. It is thus crucial that the casting process is performed with matter of precision, as the mould might exhibit a misalignment in crystal orientation, and this could later lead to unexpected mechanical properties or failure.

Since single-crystal materials are anisotropic, the crystallographic orientation will significantly influence the mechanical properties, such as yielding [16, 17], low-cycle fatigue (LCF) [18, 19], fatigue-creep interaction [20, 21], and creep [22]. During strain-controlled fatigue, materials with a low stiffness are superior, and pure nickel has the following stiffness in the nominal main crystallographic orientations: $E_{\langle 001\rangle}=125 \mathrm{GPa}, E_{\langle 011\rangle}=220 \mathrm{GPa}$ and $E_{\langle 111\rangle}=294 \mathrm{GPa}$ [12]. Even though gas turbine blades are casted with the [001] direction upwards, 
properties in the other main orientations may be of interest. For example, crack propagation is sometimes observed on the blade platform and this propagation is significantly influenced by the properties in other crystal orientations, such as the [011] direction, or in a notch where a multiaxial state is present. Studies concerning the crystal orientation influence on the TMF behaviour are rarely seen in the literature, however it has been shown that the [001] crystallographic direction shows longer TMF life compared to the [011] direction when similar mechanical strain ranges were compared [23].

From an industrial perspective in designing gas turbines, concerning the fatigue life, it is of great significance that the proper behaviour is predicted in an FEsimulation. As gas turbine blades are casted with the longitudinal direction parallel to the nominal [001] crystal orientation within an error margin of less than $10^{\circ}$, thus the misalignment need to be addressed in an FE-analysis to be able to fully capture the effect of the misalignment. Furthermore, it has been shown by FE-simulations that the variation in primary and secondary crystal orientations influence the fatigue life [24], where the secondary crystal orientation from the casting process had a pronounced effect on the LCF life of a single-crystal turbine blade. Moreover, the effect of aligning the secondary crystal orientation to the notch of a test specimen and evaluating the LCF life through FE-simulations was investigated in [25]. The influence of crystal orientation on a single-crystal turbine blade was evaluated in [26], and the conclusion drawn was that the deterministic approaches for fatigue life assessment is only valid in a certain crystal orientation. In addition, it has been shown, through FE-simulations, that the misalignment from the axial direction of the crystal orientation has a significant effect on the vibration characteristics of a turbine blade made of the single-crystal nickel-base superalloy DD6 [27].

It is well recognised that, during strain-controlled fatigue, the fatigue life of a single-crystal superalloy is highly dependent on the crystal orientation due to their anisotropic elastic properties, see e.g. [28] regarding a LCF loading condition. There it was shown that when the elastic stiffness is taken into account, the 
influence from the crystal orientation on the fatigue life almost disappears. The aim of this study is to investigate this concept from a TMF perspective; does the material stiffness solemnly influence the TMF life or are there other factors that may influence the TMF life? Based on this, out-of-phase (OP) TMF testing of STAL-15, which is a recently developed high chromium containing single-crystal nickel-base superalloy with excellent oxidation/corrosion properties aiming for land-based gas turbine applications [29, 30, 31], was conducted. In addition, the aspects in dealing with the crystal orientation dependence in an FE-context are also accounted for.

\section{Experiments}

\subsection{Methods}

The single-crystal nickel-based superalloy STAL-15, with chemical composition $\mathrm{Ni}, 4.5 \mathrm{Al}, 4.9 \mathrm{Co}, 15.6 \mathrm{Cr}, 0.1 \mathrm{Hf}, 1.0 \mathrm{Mo}, 8.1 \mathrm{Ta}$ and $0.25 \mathrm{Si}$ in wt. $\%$ was considered in this study. The material was solution heat treated for $5 \mathrm{~h}$ at $1300{ }^{\circ} \mathrm{C}$ before a two-stage ageing process at $1100{ }^{\circ} \mathrm{C}$ for $6 \mathrm{~h}$ followed by $850{ }^{\circ} \mathrm{C}$ for $20 \mathrm{~h}$ was performed. Smooth TMF specimens with an approximate diameter of $6.35 \mathrm{~mm}$ and a parallel length of $25 \mathrm{~mm}$ were machined from cast bars. An Instron servohydraulic TMF machine with induction heating and forced air cooling was used, where the machine was carefully aligned prior testing to prevent buckling of the specimens. Thermocouples were spot-welded on the specimens to control the temperature. A strain-controlled OP TMF cycle ranging from $100-850{ }^{\circ} \mathrm{C}$ at $R_{\varepsilon}=-\infty$ with a heating rate of $5{ }^{\circ} \mathrm{C} / \mathrm{s}$ was used with a 5 min dwell time applied at maximum temperature during each cycle, see Fig. 1. A high-temperature extensometer with a gauge length of $12.5 \mathrm{~mm}$ was used to control the mechanical strain ranges which were chosen in order to obtain realistic fatigue lives compared to real gas turbine blades. In total, twelve TMF tests were performed in this study, see Table 1 for further details regarding the testing conditions and experimentally obtained data. 
Table 1: Material data of the TMF test specimens.

\begin{tabular}{cccccccc}
\hline Group & Specimen & $\Delta \varepsilon_{\text {mech }} \%$ & $\Delta \varepsilon_{\text {in }} \%$ & $\theta^{\circ}$ & $\phi^{\circ}$ & $E_{\text {exp }} \mathrm{GPa}$ & $N_{f}$ \\
\hline & $\mathrm{A}$ & 1.0 & 0.018 & 3.9 & 3.0 & 118.1 & 477 \\
& $\mathrm{~B}$ & 1.1 & 0.051 & 1.3 & 5.7 & 109.8 & 515 \\
& $\mathrm{C}$ & 0.9 & 0.0045 & 1.0 & 33.0 & 114.5 & 1346 \\
& $\mathrm{D}$ & 1.2 & 0.099 & 3.2 & 33.4 & 101.5 & 428 \\
& $\mathrm{E}$ & 1.24 & 0.105 & 2.0 & 23.4 & 105.6 & 255 \\
& $\mathrm{~F}$ & 1.31 & 0.179 & 1.9 & 25.4 & 109.5 & 223 \\
\hline & $\mathrm{G}$ & 0.6 & 0.063 & 38.8 & 32.8 & 245.2 & 224 \\
& $\mathrm{H}$ & 0.5 & 0.005 & 31.4 & 0.1 & 191.6 & 696 \\
& $\mathrm{I}$ & 0.45 & 0.017 & 40.2 & 33.3 & 234.8 & 490 \\
& $\mathrm{~J}$ & 0.35 & 0.004 & 42.6 & 26.7 & 233.1 & 1098 \\
& $\mathrm{~K}$ & 0.64 & 0.096 & 40.3 & 22.0 & 208.7 & 36 \\
& $\mathrm{I}$ & 0.33 & 0.007 & 41.4 & 28.1 & 234.6 & 6380 \\
\hline
\end{tabular}

The crystallographic orientation (load direction), in accordance with the stereographic triangle, that the respective specimens have can be seen in Fig. 2. Two main groups of specimens can be observed; one close to the [001] corner of the stereographic triangle (A-F) and another cluster of specimens between the [011] and [111] corners (G-L), henceforth called group 1 and group 2. The crystal orientation of the respective specimen was determined using x-ray diffraction and is quantified by the primary and secondary crystallographic orientation angles, $\theta$ and $\phi$. Since only smooth test specimens were investigated, no regard was taken to the direction of the secondary crystal orientation as it has no influence on the observed fatigue behaviour. The experimental moduli of elasticity $E_{\text {exp }}$ was measured between $100{ }^{\circ} \mathrm{C}$ and $250{ }^{\circ} \mathrm{C}$ during the first TMF cycle for each specimen. The number of cycles to failure $N_{f}$ was determined as a global load-drop of $10 \%$ from the trend line of the maximum tensile stress occurring in each cycle. The trend line was adopted to the maximum tensile stress curve between $30 \%$ and $70 \%$ of the number of cycles until the test was terminated, 
Table 2: The elastic stiffness constants of STAL-15, units in GPa.

\begin{tabular}{ccccc}
\hline Temp. ${ }^{\circ} \mathrm{C}$ & $C_{11}$ & $C_{12}$ & $C_{44}$ & $C_{11}-C_{12}$ \\
\hline 20 & 234.6 & 147.9 & 123.2 & 111.3 \\
100 & 231.8 & 147.1 & 120.1 & 111.7 \\
200 & 228.1 & 146.1 & 116.2 & 111.9 \\
300 & 224.3 & 145.2 & 112.4 & 112.0 \\
400 & 220.4 & 144.4 & 108.6 & 111.8 \\
500 & 216.3 & 143.5 & 104.9 & 111.5 \\
600 & 212.1 & 142.8 & 101.2 & 110.9 \\
700 & 207.8 & 142.0 & 97.6 & 110.2 \\
800 & 203.3 & 141.3 & 94.0 & 109.3 \\
900 & 198.7 & 140.7 & 90.5 & 108.1 \\
1000 & 193.9 & 140.1 & 87.1 & 106.8 \\
$100-250$ & 229.0 & 146.4 & 117.2 & 111.8 \\
\hline
\end{tabular}

where the termination criterion was defined as $60 \%$ load-drop, see Fig. 3 .

The elastic stiffness constants of STAL-15 have been estimated from resonance measurements [32]. Furthermore, the elastic stiffness constants are almost linear dependent with respect to temperature, and it is thus reasonable to determine a set of linear interpolated constants for the range of $100-250{ }^{\circ} \mathrm{C}$, as the experimental modulus of elasticity have been evaluated in the same range. The estimated and interpolated constants can be found in Table 2.

After the performed TMF tests, all specimens were investigated by stereomicroscopy before they were cut parallel to the loading direction for further investigation by scanning electron microscopy (SEM). The SEM samples were prepared by mechanical grinding and polishing, and no samples were etched. For the SEM investigation, a Hitachi SU70 FEG-SEM with an annular backscatter detector, making it possible to produce images by electron channelling contrast imaging, was utilised. By use of an electron backscatter diffraction (EBSD) system by 
HKL Technology, orientation imaging microscopy was performed.

\subsection{TMF behaviour}

The results from the OP TMF tests can be found in Fig. 4, where a comparison of equivalent mechanical strain ranges show that specimens from group 1 display longer TMF lives compared to the specimens in group 2. This is due to the lower stiffness shown by the specimens close to the [001] corner compared to specimens along the [011] - [111] side. In Fig. 5 the inelastic strain ranges at half life are plotted versus cycles to failure. The general trend is that the larger inelastic strain range obtained results in a shorter TMF life, which is expected. The difference in TMF life between the two groups is much smaller when the inelastic strain range is considered instead of the mechanical strain range. Thus, showing that the elastic stiffness have an important role on the TMF lives of the specimens, which have also been shown earlier for LCF conditions [28, 33, 34]. However, group 1 still shows slightly better TMF life compared to group 2 meaning that even though the material stiffness is of great importance, there are also other factors that influence the TMF life.

Further, Fig. 6 shows the peak stresses at half life versus number of cycles to failure. Notable is that the stress level on the tensile side of the TMF cycle is slightly higher for specimens in group 1 compared to specimens from group 2. This result can be compared to another study, where the same type of tests were performed on the alloy CMSX-4 [23]. In that study, the [001] crystallographic direction was compared to the [011] direction and the tensile peak stress at half life was higher for [011] compared to [001], hence an opposite behaviour. Regarding the stress levels on the compressive side of the TMF cycles, they are similar for group 1 and group 2. 


\subsection{Fracture surface appearance and deformation mechanisms}

From the fractography study clear deformation bands are visible on the specimens surface and fractures have occurred along such deformation bands in a crystallographic mode, see Fig. 7. All deformation bands and fracture surfaces are consistent with slip along one of the $\{111\}$ crystallographic planes. Most specimens from group 2, tend to show a behaviour where all deformation bands as well as the fracture surfaces are oriented the same way, meaning that only one type of $\{111\}$ slip plane has been active during deformation, see Fig. 7a). On the other hand, the specimens from group 1 show deformation bands and fractures in several directions instead, meaning that the deformation in these cases have occurred along more than one of the $\{111\}$ planes, see Fig. $7 \mathrm{~b})$. Hence, in these cases several different type of $\{111\}$ slip planes were active during the deformation. Stereomicroscopy and SEM was used to determine the number of active slip planes for each specimen, and the results are displayed in Table 3. It is to be pointed out that this approach in determining the number of active slip planes is not guaranteed to find all active planes, however it gives the number of dominant slip planes. From literature it is well known that for FCCstructures the nominal [001] crystal direction have eight equivalent slip systems, the nominal [011] direction have four, while the nominal [111] direction have six equivalent slip systems. Note that an octahedral $\{111\}$ slip plane contains three slip systems. It is therefore expected that specimens from group 1, which are close to the [001] corner in the stereographic triangle, show a higher number of active slip planes during deformation compared to the specimens in group 2 , which are located in between the [011] and [111] corners of the stereographic triangle. The possibility that the number of active slip planes might be an influencing factor of the TMF life is considered in more detail in the Discussion section.

Virgin microstructure of STAL-15 consists of cuboidal $L 1_{2}$-ordered $\gamma^{\prime}$-precipitates with a size of approximately $0.4 \mu \mathrm{m}$ in a matrix of $\gamma$. The previously discussed deformation bands on the specimens surface, appear as bands with smeared 
Table 3: The number of active slip planes (dominant deformation) in each specimen.

\begin{tabular}{lcccccccccccc}
\hline Specimen & A & B & C & D & E & F & G & H & I & J & K & L \\
\hline Active slip planes & 3 & 2 & 2 & 2 & 3 & 2 & 1 & 1 & 1 & 1 & 1 & 2 \\
\hline
\end{tabular}

$\gamma^{\prime}$-precipitates within the microstructure, see Fig. 8. These bands are parallel to the fracture surface and are stretching over long distances within the specimens. It is clearly visible that the deformation from TMF cycling is localised to these deformation bands for all tested specimens. Along the fracture surface recrystallization has occurred which is probably also assisted by high temperature oxidation during the TMF cycling. Further, Fig. 9 displays an image at a higher magnification of a deformation band and it is once again visible that the previously cuboidal precipitates are smeared and elongated inside the band. For single-crystal nickel-based superalloys, the ability to resist deformation is strongly dependent on the strength of the $\gamma^{\prime}$-precipitates. Hence, if the $\gamma^{\prime}$ precipitates becomes smeared and elongated during TMF cycling, the material weakens and the risk of failure increases.

Specimens from group 1 tend to show deformation twinning within the $\gamma / \gamma^{\prime}$ microstructure in addition to the deformation bands, see Fig. 10a) and b) where a large number of twins parallel to each other can be observed. The twins cut through the strengthening $\gamma^{\prime}$-precipitates and extends over several tens of micrometers. That this really is a twinning phenomena is confirmed by orientation imaging microscopy by EBSD where a $60^{\circ}$ misorientation is detected between the twins and their surroundings, see Fig. 10c) and d) for the EBSD map and the corresponding misorientation profile. According to the EBSD data and with support from [35] it is highly probable that the twins are formed along the $\langle 112\rangle\{111\}$ slip system, which also is the usual twin system for FCC metals. Deformation twinning has previously been reported as a major deformation mechanism for other alloys during TMF $[3,4,5,6,7,23]$. However, in the case for STAL-15, deformation twinning seems to be a minor deformation mechanism. The fact that twins are only found in specimens from group 1 and 
how this may influence the TMF life is further discussed in the Discussion section.

\section{Applicability to finite element simulations}

The crystallographic orientation of the single-crystal superalloy have a profound impact on the mechanical response of a single-crystal component, as the singlecrystal superalloy is highly anisotropic. Thus, a resulting misalignment from the casting process can generate unintended mechanical behaviour that needs to be accounted for in e.g. an FE-analysis. This can surely be performed in a number of ways, see e.g. [26, 36], a different way to account for this misalignment in an FE-context is described below.

\subsection{Misalignment considerations}

One of the most common approaches is to consider a loading in either of the three nominal crystal orientations [001], [011] and [111], due to the effect these can have in a multiaxial condition, e.g. a notch, regarding the mechanical properties. Initially, consider a specimen with a perfect aligned crystal loaded in the nominal [001] crystallographic orientation, where the loading axis is e.g. alinged with the $z$-axis in the global FE-coordinate system, see Fig. 11a). From the casting process the two angles $\theta$ and $\phi$, as described in the experimental section, are quantified with respect to the nominal [001] crystal orientation. Subsequently, to achieve a [011] or a [111] crystallographic orientation in the FE-context the perfect aligned crystal is transformed with a positive rotation around the [100]-axis ( $x$-axis) with respect to $\theta$ and respectively around the [001] with the angle $\phi$, see Fig. 11b) and c). Thus, this process is used to define the crystal orientation, as precisely as possible by taking the two angles into consideration, that is present in the individual specimen with respect to the global FE-coordinate system. Hence, generating a slightly misalignment 
compared to the nominal orientation, under the circumstances that $\theta$ and $\phi$ do not coincide with a nominal crystal orientation. The response obtained from an FE-analysis and all the internally defined crystallographic entities (such as stresses and strains) in the material model will be based on this misalignment, see e.g. [37]. To take this into consideration, in quantifying all the internal crystallographic entities in the FE-model, the crystal orientation with respect to the global FE-coordinate system is thus defined as a two-step transformation, as depicted in Fig. 11, from the nominal [001] orientation according to

$$
A=Q_{2} Q_{1} X
$$

where $\boldsymbol{Q}_{1}$ is the transformation tensor with respect to $\theta$ around the [100]-axis, $\boldsymbol{Q}_{2}$ is the transformation tensor with respect to $\phi$ around the [001]-axis and $\boldsymbol{X}$ is a tensor that contain the nominal crystal orientation [001] aligned with the global FE-coordinate system. The outcome $\boldsymbol{A}$ contain the crystal orientation, in which the first row correspond to the [100] crystal orientation and the second to the [010] crystal orientation defined in the global FE-coordinate system, thus incorporating the misalignment according to $\theta$ and $\phi$. These two rows, named $\boldsymbol{a}_{1}$ and $\boldsymbol{a}_{2}$, constitutes the crystal orientation vectors which are input parameters to an FE-simulation. The third crystal orientation vector $\boldsymbol{a}_{3}$ (third row) can also be extracted and used as input, but as the three are mutually orthogonal the third can be obtained by regular cross product in the material model. These vectors, based on the misalignment, are then used to define all matter of crystallographic related entities in a material model, such as crystallographic shear stresses and the elastic stiffness.

\subsection{Anisotropic elastic stiffness}

As previously mentioned single-crystal superalloys are elastically anisotropic, and an important objective is to be able to describe the corresponding elastic stiffness relative to the loading direction in an FE-simulation correctly. Thus, 
the elastic behaviour of the single-crystal superalloy using an anisotropic elastic tangent stiffness tensor, as defined in [37] based on [38], has been adopted. The anisotropic elastic tangent stiffness tensor consists of two parts, one isotropic and one anisotropic, where the latter is dependent on the structural tensors $\boldsymbol{M}_{1}$ and $\boldsymbol{M}_{2}$. These are composed of the respective crystal orientation vector $\boldsymbol{a}_{1}$ and $\boldsymbol{a}_{2}$ through a dyadic product according to

$$
\begin{aligned}
& M_{1}=a_{1} \otimes a_{1} \\
& M_{2}=a_{2} \otimes a_{2}
\end{aligned}
$$

The following form of the elastic stiffness tensor was used

$$
\begin{aligned}
\mathbb{C}^{e}= & \lambda \boldsymbol{I} \otimes \boldsymbol{I}+\mu(\boldsymbol{I} \otimes \underline{I}+\boldsymbol{I} \bar{\otimes} \boldsymbol{I})+2 \eta\left(\boldsymbol{M}_{1} \otimes \boldsymbol{M}_{1}+\boldsymbol{M}_{2} \otimes \boldsymbol{M}_{2}\right. \\
& \left.+\boldsymbol{M}_{1} \otimes \boldsymbol{M}_{2}-\boldsymbol{I} \otimes \boldsymbol{M}_{1}-\boldsymbol{I} \otimes \boldsymbol{M}_{2}\right)_{m s}
\end{aligned}
$$

where $\lambda, \mu$ are the Lamé constants, $\eta$ is an additional third elastic constant, and where the subscript $m s$ denotes major symmetry. Furthermore, $\boldsymbol{I}$ is the unit tensor and the presented dyadic products are defined using standard index notation as $(\boldsymbol{a} \otimes \boldsymbol{b})_{i j}=a_{i} b_{j},(\boldsymbol{A} \otimes \boldsymbol{B})_{i j k l}=A_{i j} B_{k l},(\boldsymbol{A} \bar{\otimes} \boldsymbol{B})_{i j k l}=A_{i k} B_{j l}$ and $(\boldsymbol{A} \underline{\otimes} \boldsymbol{B})_{i j k l}=A_{i l} B_{j k}$. It is to be pointed out that Voigt notation has been applied throughout the calculations of the stiffness tensor for simplicity reasons. The use of structural tensors is a way to mathematically describe a prevailing anisotropy due to an existing internal structure in the material, in this case the crystallographic orientation. It can be shown, see [12], that the elastic stiffness tensor in Voigt notation has the following appearance for a nominally loaded [001] oriented crystal using the elastic stiffness constants presented in Table 2

$$
\mathbb{C}^{e}=\left[\begin{array}{cccccc}
C_{11} & C_{12} & C_{12} & 0 & 0 & 0 \\
C_{12} & C_{11} & C_{12} & 0 & 0 & 0 \\
C_{12} & C_{12} & C_{11} & 0 & 0 & 0 \\
0 & 0 & 0 & C_{44} & 0 & 0 \\
0 & 0 & 0 & 0 & C_{44} & 0 \\
0 & 0 & 0 & 0 & 0 & C_{44}
\end{array}\right]
$$


Table 4: The elastic constants for the investigated temperature interval, units in GPa.

\begin{tabular}{cccc}
\hline Temp. ${ }^{\circ} \mathrm{C}$ & $\lambda$ & $\mu$ & $\eta$ \\
\hline $100-250$ & -5.4 & 117.2 & -151.8 \\
\hline
\end{tabular}

Thus, by applying a nominal [001] crystal orientation $(\theta=0$ and $\phi=0)$ in Equation (1) and calculating the respective structural tensor, and subsequently inserting these into the elastic stiffness tensor expression, the three elastic constants defined in Equation (4) can be received by comparison of Equation (5), hence

$$
\left\{\begin{array}{l}
\lambda=C_{11}-2 C_{44} \\
\mu=C_{44} \\
\eta=C_{11}-C_{12}-2 C_{44}
\end{array}\right.
$$

Based on these expressions and the interpolated values presented in Table 2, the three elastic constants can be determined for the investigated temperature interval as seen in Table 4. It is to be pointed out that these parameters are temperature dependent and yields the desired properties of the stiffness tensor during a TMF cycle. Furthermore, as no temperature gradients are present in the context of a homogeneous smooth test specimen, the need for thermal expansion is not necessary.

\subsection{Uniaxial moduli of elasticity}

The uniaxial response was investigated to evaluate the uniaxial moduli of elasticity in the corresponding crystal orientations. The basic elastic relationship between stress and strain follows

$$
\sigma=\mathbb{C}^{e}: \varepsilon
$$

and to obtain the uniaxial moduli of elasticity a uniaxial loading was applied in the $z$-direction. Thus, with the corresponding stress tensor in Voigt notation $\sigma=\left[\begin{array}{cccccc}0 & 0 & \sigma_{z} & 0 & 0 & 0\end{array}\right]^{T}$ we receive, through the inverse relationship 
of Equation (7), the strain in the $z$-direction due to the configuration of the anisotropic elastic stiffness tensor. Furthermore, by assuming uniaxial elasticity we can presume that Hooke's law is valid and that the following holds

$$
E_{\text {num }}=\frac{\sigma_{z}}{\varepsilon_{z}}
$$

where $E_{\text {num }}$ is the numerically determined moduli of elasticity for the temperature interval in question, and it corresponds to $\mathbb{C}^{e}(3,3)$ which have different properties due to the crystal orientation that is present. The representation of the different uniaxial moduli of elasticity that is obtain for the different crystal orientations can be seen in Fig. 12, where all the crystal orientations in the stereographic triangle are visualised. Here, it is obvious that orientations near the nominal [001] crystallographic orientation experience the lowest stiffness, the opposite is true for orientations near the nominal [111] crystallographic orientation, and orientations near the nominal [011] crystallographic orientation lie in between, but closer to the [111] than the [001] orientation. The respective uniaxial moduli of elasticity for the test specimens, determined by the numerical procedure above, and the ratios compared to the experimental value $E_{\text {num }} / E_{\text {exp }}$ can be found in Table 5. The ratios are visualised in Fig. 13, and as can be seen, the procedure to quantify the numerical moduli of elasticity gives values that do not scatter as much as the experimental ones.

During the experiments it was derived from the mechanical strain range and the inelastic strain range, depict in Fig. 4 and 5, that the elastic stiffness influence the TMF life. To further investigate the influence of the elastic stiffness on the TMF life, the mechanical strain range was weighted by the respective numerical moduli of elasticity and plotted versus the number of cycles to failure, see Fig. 14. A curve fit was made for each of the two groups, and once again, $c f$. Fig. 5, it is visible that the difference between the two groups is much smaller when the elastic stiffness is taken into consideration. However, there is still a difference between the groups which strengthens the statement that other factors than that the elastic stiffness solemnly influence the TMF life, e.g. the number of 
Table 5: The numerical uniaxial moduli of elasticity and ratio compared to the experiments for the analysed temperature interval.

\begin{tabular}{ccc}
\hline Specimen & $E_{\text {num }} \mathrm{GPa}$ & $E_{\text {num }} / E_{\text {exp }}$ \\
\hline $\mathrm{A}$ & 116.4 & 0.9855 \\
$\mathrm{~B}$ & 115.0 & 1.0475 \\
$\mathrm{C}$ & 114.9 & 1.0039 \\
$\mathrm{D}$ & 115.9 & 1.1417 \\
$\mathrm{E}$ & 115.2 & 1.0914 \\
$\mathrm{~F}$ & 115.2 & 1.0522 \\
$\mathrm{G}$ & 238.3 & 0.9719 \\
$\mathrm{H}$ & 187.2 & 0.9773 \\
$\mathrm{I}$ & 244.5 & 1.0414 \\
$\mathrm{~J}$ & 243.9 & 1.0462 \\
$\mathrm{~K}$ & 229.8 & 1.1009 \\
$\mathrm{~L}$ & 242.5 & 1.0335 \\
\hline
\end{tabular}

active slip planes.

\subsection{Crystallographic resolved shear stress}

During plastic flow in a single-crystal slip will occur along the slip planes, which induce shear stresses. If enough slip is present so called persistent slip bands will appear [39], and as could be seen in the microscopy observations the specimens exhibit deformation bands across the surface. When a slip plane is plastically deformed it is said to be active, and slip might be located on one or several type of $\{111\}$ planes. Moreover, it has been shown that these crystallographic slip planes may act as crack initiation points $[25,40,41]$ that may lead to failure of the component. Hence, the active slip planes which the main deformation occurs on is interesting from a failure perspective, and to be able to observe a comparable behaviour between the experiments and FE-simulations the crystallographic resolved shear stresses on the slip planes are evaluated. 
When a material flows plastically it usually hardens due to accumulation of dislocations on the crystallographic slip planes. Work hardening might be present in single-crystal materials, e.g. copper and aluminium, and can be described by using a work hardening matrix able to quantify the self-hardening and latenthardening effects of the crystal, see e.g. [42] regarding modelling of work hardening in single-crystals. However, in single-crystal nickel-base superalloys there is typically very little work hardening, see e.g. [37], as the strengthening $\gamma^{\prime}$ precipitates effectively stops the dislocation movement [43, 44]. Furthermore, it is to be noted that the dominant deformation mechanism occurring in the material investigated in this paper, STAL-15, is due to slip. Thus, the twinning mechanism is of less concern regarding the damaging effect it has on the TMF behaviour. In the single-crystal nickel-base superalloys CMSX-4 and MD2 TMF damage is, on the contrary, attributed to twinning [3,45]. The ambition here is to evaluate the damaging mechanism in the material during TMF and not to evaluate and describe/model the constitutive behaviour of the material. Hence, no evolution and coupling of twinning and slip resistance have been accounted for in the present study, a general description of this coupling can e.g. be found in [46].

Based on the framework derived earlier in quantifying the misalignment in the specimens, and how these are taken care of in an FE-analysis, the resolved shear stress on all crystallographic octahedral slip systems $(\alpha=1,2, \ldots, 12)$ of the specimens are numerically quantified by applying a tensile load to investigate if the slip system is active or not. The process is performed by a simple projection of a tensile load, in the $z$-direction, down on the respective slip plane $\boldsymbol{n}^{\alpha}$ and direction $s^{\alpha}$ using a Fortran script. The procedure is based on the crystal plasticity model developed in [37], which later was expanded to incorporate creep relaxation under TMF loading conditions [47]. Regard is only taken to the resolved shear stress which acts along the slip direction (Burgers vector) that the crystal might plastically deformed in, other non-Schimd stresses are of course essential to describe the macroscopic behaviour of the single-crystal 
Table 6: Crystallographic direction vectors of the resolved shear stress.

\begin{tabular}{ccc}
\hline$\alpha$ & $\boldsymbol{n}^{\alpha}$ & $\boldsymbol{s}^{\alpha}$ \\
\hline 1 & $(111)$ & {$[01 \overline{1}]$} \\
2 & $(111)$ & {$[\overline{1} 01]$} \\
3 & $(111)$ & {$[1 \overline{1} 0]$} \\
4 & $(1 \overline{1} \overline{1})$ & {$[0 \overline{1} 1]$} \\
5 & $(1 \overline{1} \overline{1})$ & {$[\overline{1} 0 \overline{1}]$} \\
6 & $(1 \overline{1} \overline{1})$ & {$[110]$} \\
7 & $(\overline{1} 1 \overline{1})$ & {$[011]$} \\
8 & $(\overline{1} 1 \overline{1})$ & {$[10 \overline{1}]$} \\
9 & $(\overline{1} 1 \overline{1})$ & {$[\overline{1} \overline{1} 0]$} \\
10 & $(\overline{1} \overline{1} 1)$ & {$[0 \overline{1} \overline{1}]$} \\
11 & $(\overline{1} \overline{1} 1)$ & {$[101]$} \\
12 & $(\overline{1} \overline{1} 1)$ & {$[\overline{1} 10]$} \\
\hline
\end{tabular}

superalloy, but these are not considered here. The crystallographic direction vectors, $\boldsymbol{n}^{\alpha}$ and $\boldsymbol{s}^{\alpha}$, which the tensile load is projected on can be seen in Table 6 . It is to be noted that the crystallographic direction vectors are composed of the three crystal orientation vectors $\boldsymbol{a}_{1}, \boldsymbol{a}_{2}$ and $\boldsymbol{a}_{3}$, where e.g. [101] is represented by $\left[1 \cdot \boldsymbol{a}_{1}+0 \cdot \boldsymbol{a}_{2}-1 \cdot \boldsymbol{a}_{3}\right]$, hence addressing the misalignment throughout the procedure. This is also true in an FE-context, where the crystal orientation vectors are input parameters. The projection is performed in accordance with

$$
\tau^{\alpha}=\boldsymbol{s}^{\alpha} \cdot \boldsymbol{\sigma} \cdot \boldsymbol{n}^{\alpha}
$$

For the respective specimen the script then normalises the calculated resolved shear stresses with regard to the maximum resolved shear stress obtained on one of the slip systems in that specimen. A comparison to nominally oriented specimens, hence [001], [011] and [111] crystal orientations, have also been performed, as in which eight, four and six slip systems are active respectively. The 
normalised resolved shear stresses and the maximum values due to a tensile load of $10 \mathrm{MPa}$ in the $z$-direction can be found in Table 7 . For the normalised stresses, unity represents the slip system(s) that attained the maximum value(s), thus an active slip system. Further, it is to be pointed out that some values are very close to unity, this/these slip system(s) are active but not the one which the main deformation occurs along. All responses are given in absolute, as a negative resolved shear stress only correspond to a negative direction compared to the slip direction in the above projection. Another aspect is that a slip plane contain three slip systems, and it is vital to observe which slip systems that are active in correspondence to the respective slip plane. It might be that two active slip systems are located on the same plane, thus only one active slip plane is received.

In Table 7 attention is drawn to that the maximum value of the shear stress is received in the specimen with few active slip systems consisting of peak stresses versus specimens with lower shear stresses more evenly distributed on several slip systems. The intermutual comparison (normalisation) of the shear stresses on all the slip systems are performed to define a comparative measure of the shear stresses if the system is active or not. In this work the comparative measure of $1-0.9$ defines an active system, $0.89-0.7$ constitutes nearly active systems and those that are less are not active. One can trail back to the basic crystal plasticity yield criterion, in which the resolved shear stress on a slip system flows plastically if a critical resolved shear stress is reached (Schimd's law), see e.g. [48]. Thus, it is reasonable to assume that a system with a comparative measure of about 1 is active. Consider specimen $\mathrm{H}$, which has the highest shear stress of all the specimens, with two active slip systems (1.0 and 0.999) and all other slip systems are experiences considerably less shear stress. The next is specimen $\mathrm{K}$, which has one active system and two system close to be active (0.838 and 0.742 ), followed by L, G, J and I with decreasing maximum shear stresses and more evenly distributed stresses in stages. Finally, group 1 follows with even smaller shear stresses and thus more active slip systems. Furthermore, it is also 
Table 7: The normalised and the maximum resolved shear stress of all slip systems for each specimen and the nominal [001], [011] and [111] crystal orientations.

\begin{tabular}{|c|c|c|c|c|c|c|c|c|c|c|c|c|c|c|c|}
\hline$\alpha$ & & & & & & & & $\tau^{\alpha}$ & & & & & & & \\
\hline & {$[001]$} & [011] & {$[111]$} & $\mathrm{A}$ & B & $\mathrm{C}$ & $\mathrm{D}$ & $\mathrm{E}$ & $\mathrm{F}$ & $\mathrm{G}$ & $\mathrm{H}$ & I & $\mathrm{J}$ & $\mathrm{K}$ & $\mathrm{L}$ \\
\hline 1 & 1.0 & 0.0 & 0.0 & 0.935 & 0.978 & 0.995 & 0.981 & 0.981 & 0.983 & 0.384 & 0.390 & 0.351 & 0.200 & 0.232 & 0.253 \\
\hline 2 & 1.0 & 1.0 & 0.0 & 0.999 & 0.999 & 0.999 & 0.997 & 0.999 & 0.999 & 0.669 & 0.999 & 0.640 & 0.659 & 0.742 & 0.665 \\
\hline 3 & 0.0 & 1.0 & 0.0 & 0.065 & 0.020 & 0.005 & 0.016 & 0.018 & 0.016 & 0.285 & 0.610 & 0.289 & 0.459 & 0.510 & 0.412 \\
\hline 4 & 1.0 & 0.0 & 0.0 & 0.929 & 0.975 & 0.976 & 0.925 & 0.955 & 0.957 & 0.226 & 0.389 & 0.201 & 0.126 & 0.162 & 0.157 \\
\hline 5 & 1.0 & 1.0 & 1.0 & 1.0 & 1.0 & 1.0 & 1.0 & 1.0 & 1.0 & 1.0 & 1.0 & 1.0 & 1.0 & 1.0 & 1.0 \\
\hline 6 & 0.0 & 1.0 & 1.0 & 0.071 & 0.025 & 0.024 & 0.075 & 0.045 & 0.044 & 0.774 & 0.611 & 0.799 & 0.874 & 0.838 & 0.843 \\
\hline 7 & 1.0 & 0.0 & 1.0 & 0.935 & 0.980 & 0.995 & 0.984 & 0.982 & 0.984 & 0.715 & 0.391 & 0.711 & 0.542 & 0.491 & 0.588 \\
\hline 8 & 1.0 & 0.0 & 0.0 & 0.873 & 0.956 & 0.971 & 0.911 & 0.938 & 0.942 & 0.241 & 0.242 & 0.223 & 0.175 & 0.187 & 0.193 \\
\hline 9 & 0.0 & 0.0 & 1.0 & 0.063 & 0.024 & 0.024 & 0.073 & 0.044 & 0.042 & 0.474 & 0.148 & 0.488 & 0.367 & 0.303 & 0.395 \\
\hline 10 & 1.0 & 0.0 & 1.0 & 0.928 & 0.975 & 0.976 & 0.922 & 0.954 & 0.956 & 0.105 & 0.388 & 0.160 & 0.215 & 0.096 & 0.178 \\
\hline 11 & 1.0 & 0.0 & 1.0 & 0.872 & 0.956 & 0.971 & 0.908 & 0.937 & 0.941 & 0.090 & 0.241 & 0.137 & 0.167 & 0.071 & 0.142 \\
\hline 12 & 0.0 & 0.0 & 0.0 & 0.056 & 0.019 & 0.005 & 0.014 & 0.017 & 0.015 & 0.015 & 0.147 & 0.023 & 0.048 & 0.025 & 0.036 \\
\hline$\tau_{\max }^{\alpha} \mathrm{MPa}$ & 4.082 & 4.082 & 2.722 & 4.341 & 4.173 & 4.141 & 4.262 & 4.209 & 4.201 & 4.415 & 4.792 & 4.332 & 4.402 & 4.595 & 4.429 \\
\hline
\end{tabular}


to be noted that specimen $\mathrm{H}$ is the most divergent oriented specimen in group 2, which might be a reason for the difference in active slip systems.

\section{Discussion}

The objective of this paper was to study the crystal orientation influence on the TMF behaviour of a single-crystal nickel-based superalloy; both from an experimental and a modelling perspective. Experimental results show that there is a strong orientation dependence on the TMF life under strain-controlled TMF, where specimens with a low stiffness also show the longest TMF life (when equivalent mechanical strain ranges are compared), see Fig. 4. Hence, the TMF life is highly dependent on the elastic stiffness, which have to be considered due to the elastic anisotropy shown by single-crystal superalloys [18, 28]. Furthermore, this study reveals that the number of active slip planes in the material may also be one factor which influence the TMF life. In the case where several type of $\{111\}$ slip planes are active a longer TMF life, with respect to the inelastic strain range, is found compared to the case where only one slip plane is active, see Fig. 5. Several slip planes result in a widespread deformation where the amount of plastic deformation within each deformation band is relatively low, which is good since crack growth during TMF is preferred along these bands. However, when only one slip plane is active the plastic deformation is more concentrated which seems to be detrimental for the TMF life. Even when the elastic stiffness is taken into consideration, specimens with several slip planes result in a longer TMF life, see Fig. 14. Here it is visible that the two groups merge at low strain ranges, this is expected as a result of the weighting with respect to the moduli of elasticity and the low amount of plasticity which is present. An increasing number of cycles to failure is received as the influence of the inelastic strain is diminishing, and the influence of the number of active slip planes is thus also diminishing. The material behaviour is moving towards an elastic state. This behaviour is different from what has been reported earlier regarding LCF [28], 
where the crystal orientation influence disappeared for all investigated mechanical strain ranges when the elastic stiffness was taken into account. Hence, here it seems to be a difference between LCF and TMF which might be attributed to the very localised deformation that has been shown to occur during TMF $[3,23]$. Deformation bands, with smeared and elongated $\gamma^{\prime}$-precipitates, are created during TMF cycling and the TMF life is certainly dependent on the ability to resist crack propagation along the deformation bands

Further, deformation twins are found to be one deformation mechanism occurring during TMF and the creation of twins seems to depend on in which crystallographic direction the material is loaded. The influence from twins on the TMF life has been reported earlier, see e.g. $[3,4,5,6,7,23]$, and in this study it seems that the formation of twins, see Fig. 10, is preferred compared to formation of the localised deformation bands with smeared and elongated $\gamma^{\prime}$-precipitates, see Fig. 8 and 9, when a good TMF life is desired. The reason for this is likely to be attributed to the fact that when the $\gamma^{\prime}$-precipitates are smeared and elongated, they lose their strengthening effect to a greater extent compared to when twinning occurs, where the twins certainly cut the $\gamma^{\prime}$-cuboidals but preserving their shape.

The number of active slip planes during deformation of the single-crystal agree well between the numerical and experimental observed numbers, $c f$. Table 3 and 7 . As the number of active slip planes are difficult to determine experimentally, the least number of active planes are stated and there is a possibility that more are active, but these could not be verified. In addition, the ones observed are the planes where the main deformation occurred and others might still be active but to a much lower degree. When investigating the numerically obtained resolved shear stresses it can be observed that group 1 exhibits more or less eight active slip systems (four slip planes) throughout the analyses and that group 2 show a little more erratic response, where approximately one active plane is obtained per specimen. The question arising is; at what comparative level of shear stress is the plane considered to be active? As pointed out in the previous section 
the comparative measure was defined for an active plane. One can of course discuss the levels of this measure, but it is a qualitatively measure which gives an indication of active planes with respect to the microstructural observations. Furthermore, it is to be noted that it is always slip system 5 which exhibits the maximum resolved shear stress in all of the specimens. This is due to that the procedure is numerically dependent on the crystal orientation, as that slip system might be favourable positioned after the performed transformations in comparison to the applied tensile load.

The elastic stiffness constants were estimated from resonance measurements and later used in the procedure to obtain numerically usable parameters to be applied in an FE-context. The misalignment in the specimens were considered when the respective numerical uniaxial moduli of elasticity was obtained. From the comparison of the numerical modulus of elasticity with respect to the experimental ones, obtained from the performed TMF tests, a good agreement was achieved, see Fig. 13. A possible source of error can be the estimation of the resonance measurements, and it has been the authors assumption that a nominal [001] crystal orientation has been used in the resonance measurement estimations. This might not be the case, thus giving some possible errors in the evaluation process of the elastic constants $\lambda, \mu$ and $\eta$. In addition, the experimental modulus of elasticity scatter largely, especially for group 1, and one can reason that a statistical scatter might be present in the material, arising from e.g. microstructural features.

The industrial relevance in using the above defined procedure, is that during a design process of a gas turbine component, the influence of the crystallographic orientation is easily accounted for by the two angles $\theta$ and $\phi$ obtained in the casting process. By incorporating the angles through the crystallographic orientation vectors $\boldsymbol{a}_{1}$ and $\boldsymbol{a}_{2}$, the elastic and plastic behaviour in an FE-simulation can be correctly described of the casted component. The framework defined in this study can be applied in an industrial context to investigate if a singlecrystal gas turbine component with a known misalignment from the nominal 
primary orientation [001] is acceptable to be used or not. The TMF life of the component can be quantified, as the moduli of elasticity and the number of active slip planes are predicted by the material model during an FE-simulation. A comparison to a nominal oriented component can be made to see the effects of the misalignment, and thus determining if it is acceptable or not. This can be performed on a design level, in quantifying the allowable scatter in the primary crystal orientation of the component. Thus, a more accurate fatigue response can be predicted and the industrial value, such as degree of efficiency, maintenance concern, and profit, will increase.

\section{Conclusions}

- The elastic stiffness of the material with respect to the loading direction is the strongest factor in determining the TMF life of a single-crystal nickelbased superalloy.

- However, the number of active slip planes during deformation also influences the TMF life. A higher number of active slip planes seems to be beneficial since the deformation in this case becomes more widespread. On the other hand when only one slip plane is active, the deformation is more concentrated which seems to be detrimental for the TMF life.

- Deformation twinning is a more favourable deformation mechanism compared to smeared and elongated $\gamma^{\prime}$-precipitates where the cuboidal precipitates lose their strengthening effect.

- The elastic anisotropy of the material in an FE-simulation can be qualitatively described by using the presented method based on the structural tensors of the crystal orientation.

- It was also shown that in an FE-context the number of active slip planes were correctly quantified in comparison to the microstructural observa- 
tions. A material model, capable of managing the influence of crystal orientation, is thus able to predict the behaviour of the material.

\section{Acknowledgements}

The work has been financially supported by Siemens Industrial Turbomachinery AB in Finspång, Sweden, and the Swedish Energy Agency, via the Research Consortium of Materials Technology for Thermal Energy Processes, Grant No. KME-702. In addition, the support from the Swedish Government Strategic Research Area in Materials Science on Functional Materials at Linköping University (Faculty Grant SFO-Mat-LiU \#2009 - 00971) is also acknowledged.

\section{References}

[1] N.A. Cumpsty, Jet Propulsion: A Simple Guide to the Aerodynamic and Thermodynamic Design and Performance of Jet Engines, Cambridge University Press, Cambridge, 1997.

[2] K. Dietrich, J.M. Latorre, L. Olmos, A. Ramos, The role of flexible demands in smart energy systems, in: V. Pappu, M. Carvalho, P. Pardalos (Eds.), Optimization and Security Challenges in Smart Power Grids, 2013, pp. 79-97.

[3] J.J. Moverare, S. Johansson, R.C. Reed, Deformation and damage mechanisms during thermal-mechanical fatigue of a single-crystal superalloy, Acta Materialia, 57, (2009), 2266-2276.

[4] F. Sun, J. Zhang, H. Harada, Deformation twinning and twinning-related fracture in nickel-base single-crystal superalloys during thermomechanical fatigue cycling, Acta Materialia, 67, (2014), 45-57. 
[5] J. X. Zhang, H. Harada, Y. Koizumi, T. Kobayashi, Crack appearance of single-crystal nickel-base superalloys after thermomechanical fatigue failure, Scripta Materialia, 61, (2009) 1105-1108.

[6] J. X. Zhang, H. Harada, Y. Ro, Y. Koizumi, T. Kobayashi, Thermomechanical fatigue mechanism in a modern single crystal nickel base superalloy TMS-82, Acta Materialia, 56, (2008) 2975-2987.

[7] J. X. Zhang, Y. Ro, H. Zhou, H. Harada, Deformation twins and failure due to thermo-mechanical cycling in TMS-75 superalloy, Scripta Materialia, 54, (2006) 655-660.

[8] G.M. Han, J.J. Yu, X.F. Sun, Z.Q. Hu, Thermo-mechanical fatigue behavior of a single crystal nickel-based superalloy, Materials Science and Engineering A, 528, (2011), 6217-6224.

[9] J. J. Moverare, S. Johansson, Damage mechanisms of a high-Cr single crystal superalloy during thermomechanical fatigue, Materials Science and Engineering A, 527, (2010) 553-558.

[10] F. Liu, Z. G. Wang, S. H. Ai, Y. C. Wang, X. F. Sun, T. Jin, H. R. Guan, Thermo-mechanical fatigue of single crystal nickel-based superalloy DD8, Scripta Materialia, 48, (2003) 1265-1270.

[11] P.R. Beeley, R.F. Smart, Investment Casting, Maney Materials Science, 1995.

[12] C.R. Reed, The Superalloys - Fundamentals and Applications, Cambridge University Press, Cambridge, 2006.

[13] R. Zhang, L. Chen, C. Li, N. Wang, X. Lu, Z. Ren, Influence of spiral crystal selector on crystal orientation of single crystal superalloy, Transactions of Nonferrous Metals Society of China, 22, (2012), 1092-1095.

[14] P. Carter, D.C. Cox, C.A. Gandin, R.C. Reed, Process modelling of grain selection during the solidification of single crystal superalloy castings, $\mathrm{Ma}$ terials Science and Engineering A, 280, (2000), 233-246. 
[15] S.-M. Seo, I.-S. Kim, J.-H. Lee, C.-Y. Jo, H. Miyahara, K. Ogi, Grain structure and texture evolutions during single crystal casting of the $\mathrm{Ni}$ base superalloy CMSX-4, Metals and Materials International, 15, (2009), 391-398.

[16] L.N. Wang, Y. Liu, J.J. Yu, Y. Xu, X.F. Sun, H.R. Guan, Z.Q. Hu, Orientation and temperature dependence of yielding and deformation behavior of a nickel-base single crystal superalloy, Materials Science and Engineering A, 505, (2009), 144-150.

[17] R.V. Miner, R.C. Voigt, J. Gayda, T.P. Gabb, Orientation and temperature dependence of some mechanical properties of the single-crystal nickel-base superalloy René N4: Part I. Tensile behavior, Metallurgical Transactions A, 17, (1986), 491-496.

[18] T.P. Gabb, J. Gayda, R.V. Miner, Orientation and temperature dependence of some mechanical properties of the single-crystal nickel-base superalloy René N4: Part II. Low cycle fatigue behavior, Metallurgical Transactions A, 17, (1986), 497-505.

[19] T.P. Gabb, G. Welsch, The high temperature deformation in cyclic loading of a single crystal nickel-base superalloy, Acta Metallurgica, 37, (1989), 2507-2516.

[20] D.J. Smith, L. Shu-Xin, G.E. Ellison, Influence of orientation on the fatigue and creep behavior of a single crystal nickel base superalloy, Proceedings of the Fifth International Conference on Creep of Materials, (1992), 18-21.

[21] M. Segersäll, J.J. Moverare, D. Leidermark, K. Simonsson, Creep and stress relaxation anisotropy of a single-crystal superalloy, Metallurgical and $\mathrm{Ma}$ terials Transactions A, 45, (2014), 2532-2544.

[22] J. Yu, J.R. Li, J.Q. Zhao, M. Han, Z.X. Shi, S.Z. Liu, H.L. Yuan, Orientation dependence of creep properties and deformation mechanism in DD6 
single crystal superalloy at $760{ }^{\circ} \mathrm{C}$ and $785 \mathrm{MPa}$, Materials Science and Engineering A, 560, (2013), 47-53.

[23] M. Segersäll, J. J. Moverare, K. Simonsson, S. Johansson, Deformation and damage mechanisms during thermomechanical fatigue of a single-crystal superalloy in the $\langle 001\rangle$ and $\langle 011\rangle$ directions, in: E. S. Huron, R. C. Reed, M. Hardy, M. J. Mills, R. E. Montero, P. D. Portella, J. Talesman (Eds.), Superalloys 2012, The Minerals, Metals and Materials Society, 2012, pp. $215-223$.

[24] N.K. Arakere, G. Swanson, Effect of crystal orientation on fatigue failure of single crystal nickel base turbine blade superalloys, Journal of Engineering for Gas Turbine and Power, 124, (2002), 161-176.

[25] D. Leidermark, J. Moverare, K. Simonsson, S. Sjöström, S. Johansson, Fatigue crack initiation in a notched single-crystal superalloy component, Procedia Engineering, 2, (2010), 1067-1075.

[26] M.W. Savage, The influence of crystal orienation on the elastic stresses of a single crystal nickel-based turbine blade, Journal of Engineering for Gas Turbine and Power, 134, (2012), 012501-1-012501-7

[27] Z. Wen, H. Mao, Z. Yue, B. Wang, The influence of crystal orientation on vibration characteristics of DD6 nickel-base single crystal superalloy turbine blade, Journal of Materials Engineering and Performance, 23, (2014), $372-377$.

[28] R.P. Dalal, C.R. Thomas, L.E. Dardi, The effect of crystallographic orientation on the physical and mechanical properties of an investment cast single crystal nickel-base superalloy, in: R.H. Bricknell, W.B. Kent, M. Gell, C.S. Kortovich, J.F. Radavich (Eds.), Superalloys 1984, The Minerals, Metals and Materials Society, 1984, pp. 185-197.

[29] R. C. Reed, J. J. Moverare, A. Sato, F. Karlsson, M. Hasselqvist, A new single-crystal superalloy for power generation applications, in: E. S. Huron, 
R. C. Reed, M. Hardy, M. J. Mills, R. E. Montero, P. D. Portella, J. Talesman (Eds.), Superalloys 2012, The Minerals, Metals and Materials Society, 2012, pp. 197-204.

[30] A. Sato, J. J. Moverare, M. Hasselqvist, R. C. Reed, On the mechanical behavior of a new single-crystal superalloy for industrial gas turbine applications, Metallurgical and Materials Transactions A, 43, (2012), 2302-2315.

[31] A. Sato, Y.-L. Chiu, R. Reed, Oxidation of nickel-based single-crystal superalloys for industrial gas turbine applications, Acta Materialia, 59, (2011), 225-240.

[32] Internal report, Siemens Industrial Turbomachinery AB, Finspång, Sweden, 2012.

[33] S.X. Li, E.G. Ellison, D.J. Smith, The influence of orientation on the elastic and low cycle fatigue properties of several single crystal nickel base superalloys, Journal of Strain Analysis for Engineering Design, 29, (1994), 147-153.

[34] D. Shi, J. Huang, X. Yang, H. Yu, Effects of crystallographic orientations and dwell types on low cycle fatigue and life modeling of a SC superalloy, International Journal of Fatigue, 49, (2013), 31-39.

[35] D.M. Knowles, S. Gunturi, The role of $\langle 112\rangle\{111\}$ slip in the asymmetric nature of creep of single crystal superalloy CMSX-4, Materials Science and Engineering A, 328, (2002), 223-237.

[36] N.X. Hou, W.X. Gou, Z.X. Wen, Z.F. Yue, The influence of crystal orientations on fatigue life of single crystal cooled turbine blade, Materials Science and Engineering A, 492, (2008), 413-418.

[37] D. Leidermark, J.J. Moverare, K. Simonsson, S. Sjöström, S. Johansson, Room temperature yield behaviour of a single-crystal nickel-base superalloy with tension/compression asymmetry, Computational Materials Science, 47, (2009), 366-372. 
[38] J. Schröder, F. Gruttmann, J. Löblein, A simple orthotropic finite elastoplasticity model based on generalized stress-strain measures, Computational Mechanics, 30, (2002), 48-64.

[39] S. Suresh, Fatigue of Materials, Cambridge University Press, New York, 1998.

[40] R.A. Naik, D.P. DeLuca, D.M. Shah, Critical plane fatigue modeling and characterization of single crystal nickel superalloys, Journal of Engineering for Gas Turbines and Power, 126, (2004), 391-400.

[41] D. Leidermark, M. Segersäll, J. Moverare, K. Simonsson, Modelling of TMF crack initiation in smooth single-crystal superalloy specimens, Advanced Materials Research, 891-892, (2014), 1283-1288.

[42] S. Nemat-Nasser, Plasticity: A Treatise on Finite Deformation of Heterogeneous Inelastic Materials, Cambridge University Press, Cambridge, 2004.

[43] B. Reppich, Some new aspects concerning particle hardening mechanisms in $\gamma^{\prime}$ precipitating Ni-base alloys - I. Theoretical concept, Acta Metallurgica, 30, (1982), 87-94.

[44] B. Reppich, P. Schepp, G. Wehner, Some new aspects concerning particle hardening mechanisms in $\gamma^{\prime}$ precipitating Ni-base alloys - II. Experiments, Acta Metallurgica, 30, (1982), 95-104.

[45] M. Segersäll, J.J. Moverare, D. Leidermark, S. Johansson, In- and outof-phase thermomechanical fatigue of a Ni-based single-crystal superalloy, in: J.Y. Guédou, J. Choné (Eds.), Eurosuperalloys 2014, MATEC Web of Conferences, 14, 2014, pp. 19003:1-6.

[46] J.D. Clayton, Nonlinear Mechanics of Crystals, Springer, Dordrecht, 2011.

[47] D. Leidermark, M. Segersäll, Modelling of thermomechanical fatigue stress relaxation in a single-crystal nickel-base superalloy, Computational Materials Science, 90, (2014), 61-70. 
[48] D. Peirce, R.J. Asaro, A. Needleman, An analysis of nonuniform and localized deformation in ductile single crystals, Acta Metallurgica, 30, (1982), 1087-1119.

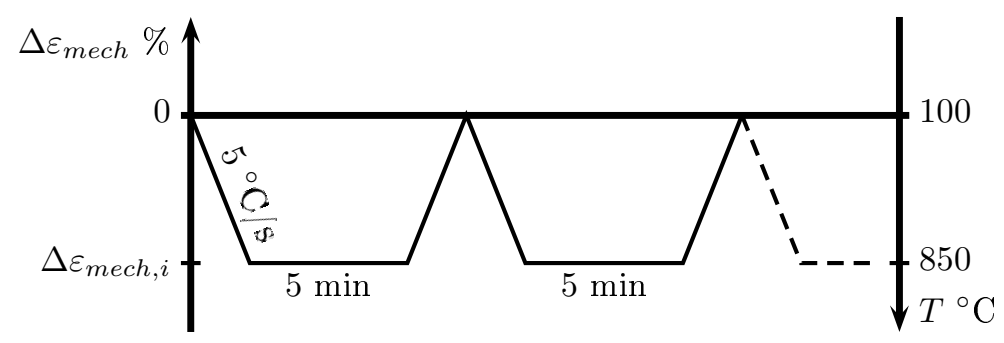

Figure 1: The OP TMF cycle which was used in the experiments of this study, where $i=$ $A, B, \ldots, L$ represents the respective tests.

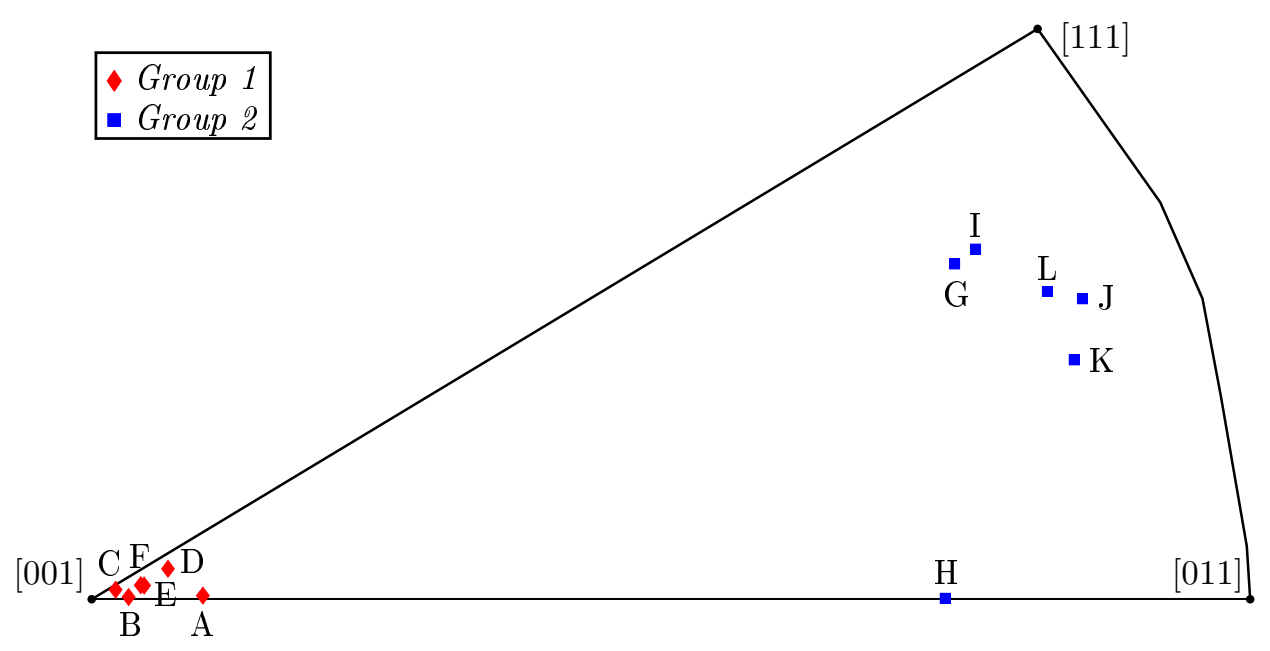

Figure 2: The orientations of the investigated test specimens in the stereographic triangle. 


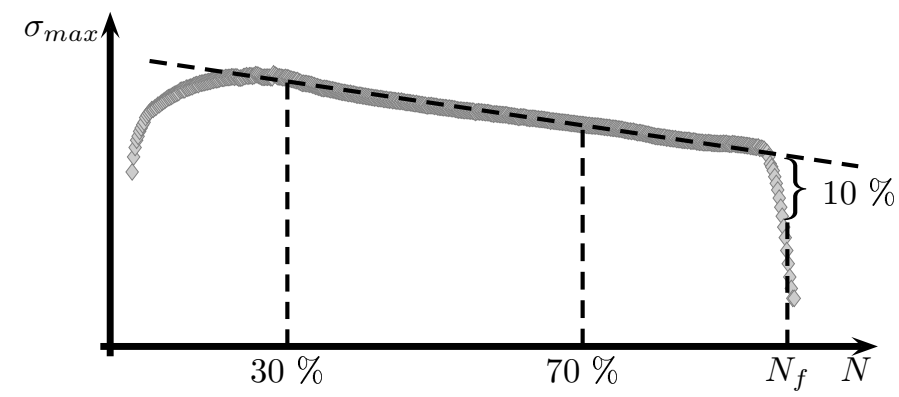

Figure 3: Evaluation of the number of cycles to failure in the $10 \%$ load-drop procedure.

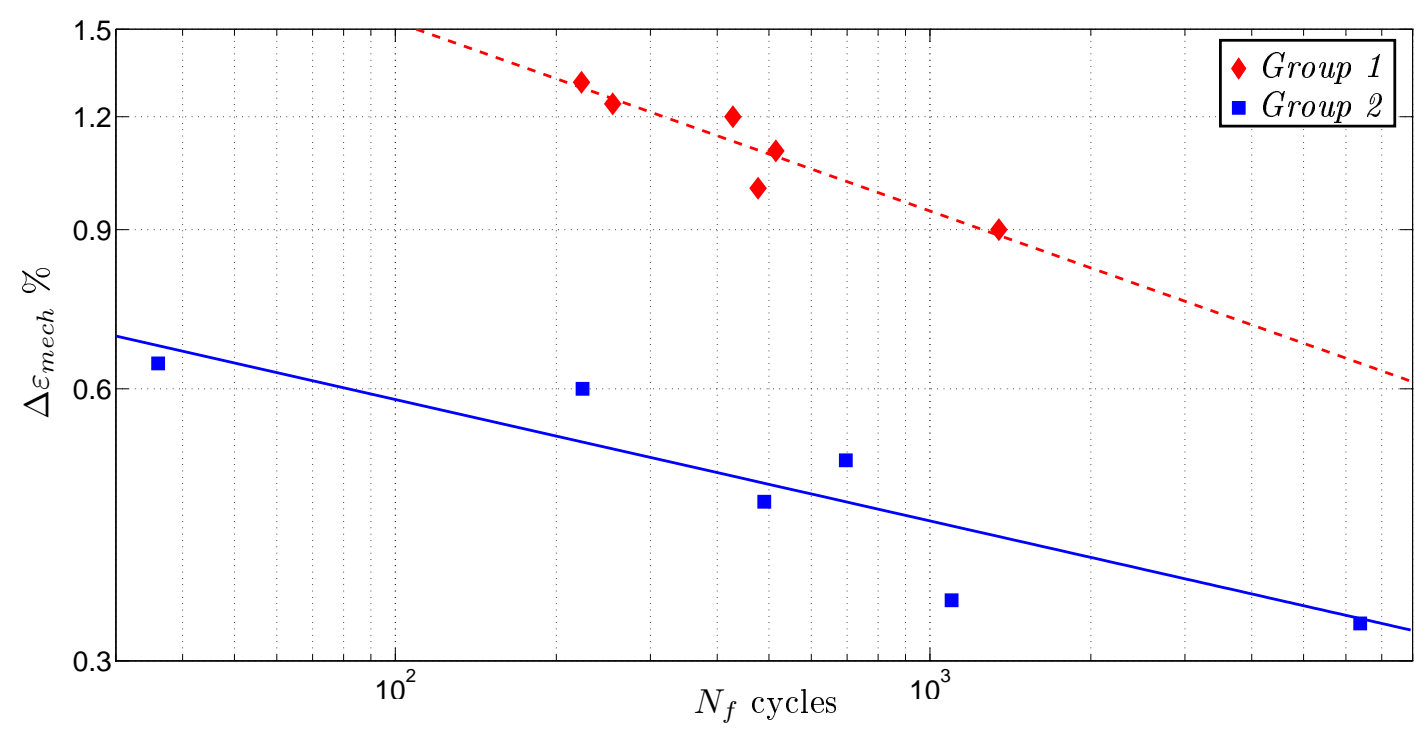

Figure 4: Mechanical strain range versus number of cycles to failure. 


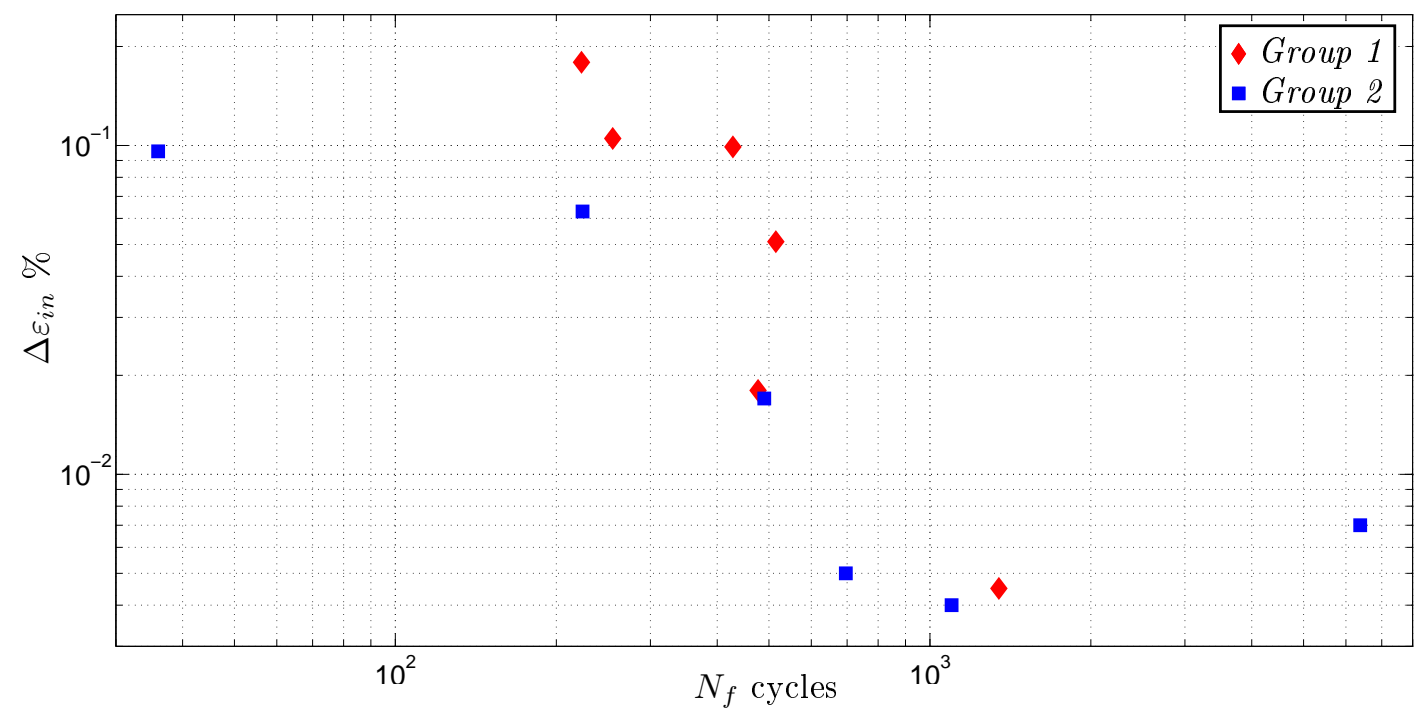

Figure 5: Inelastic strain range at half life versus number of cycles to failure.

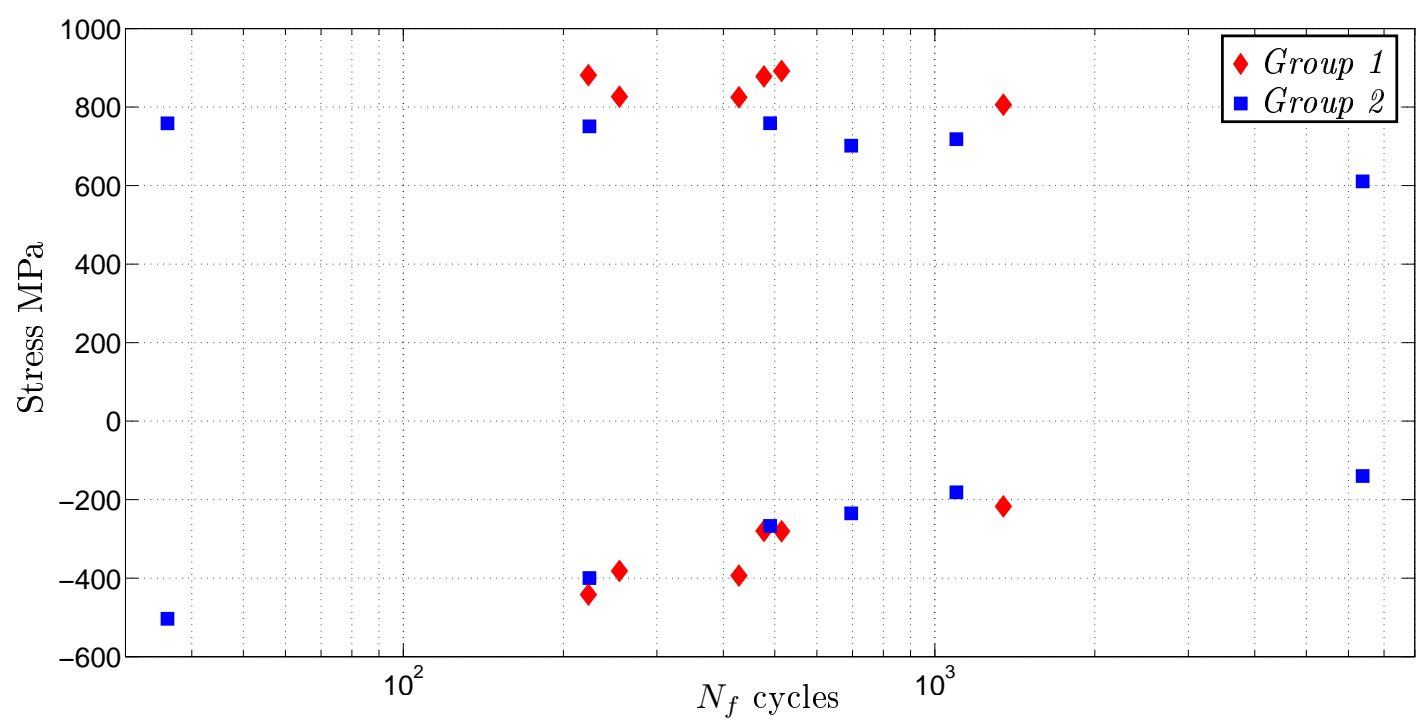

Figure 6: Peak stress at half life versus number of cycles to failure. 


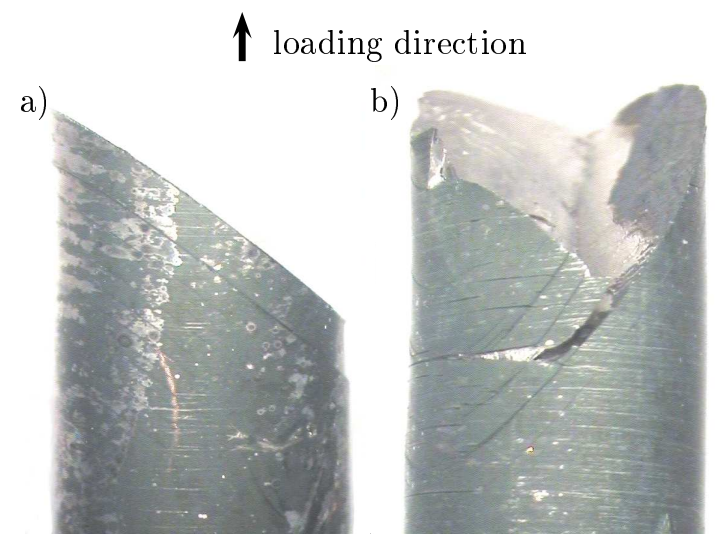

Figure 7: From fractography deformation bands are visible on the specimen surfaces, with a) one type of $\{111\}$ active slip plane and b) several different type of $\{111\}$ active slip planes.

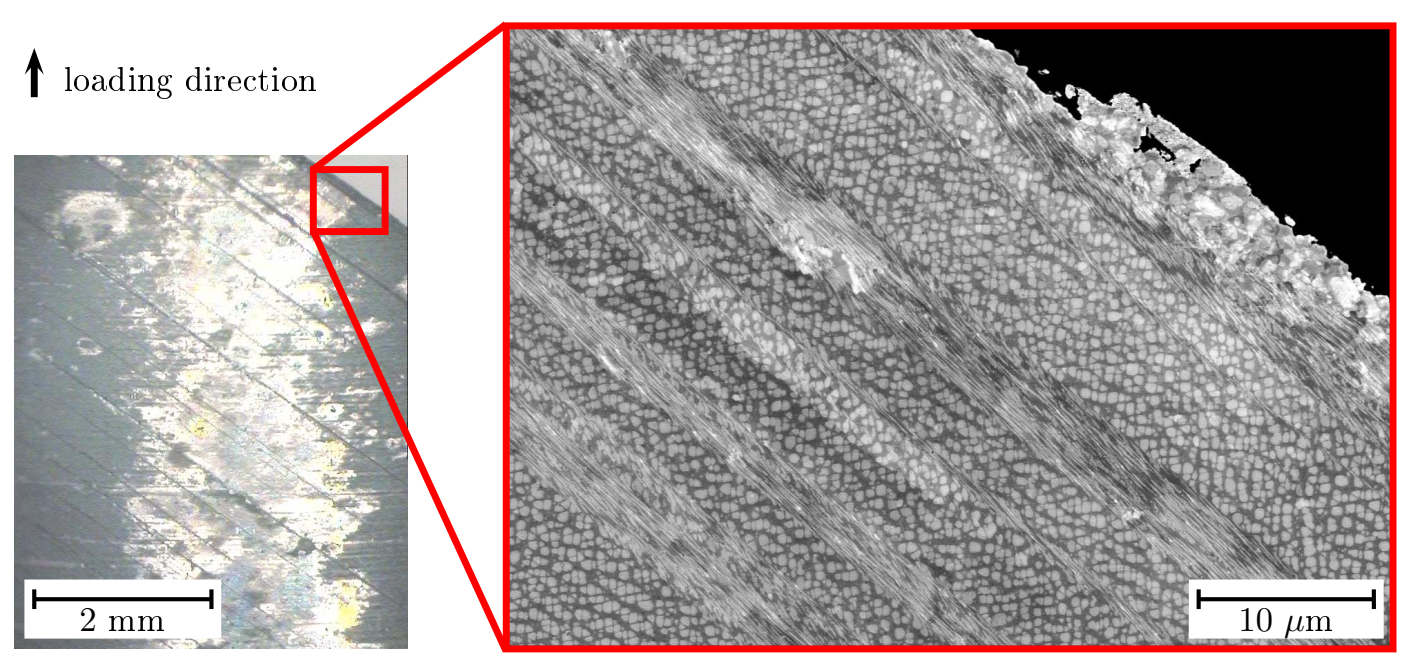

Figure 8: Deformation bands near the fracture surface of a specimen, and magnification. 


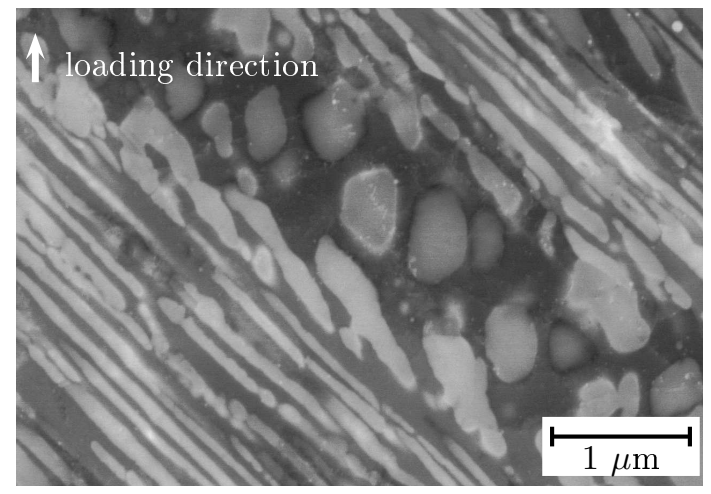

Figure 9: Smeared and elongated $\gamma^{\prime}$-precipitates within the deformation bands.
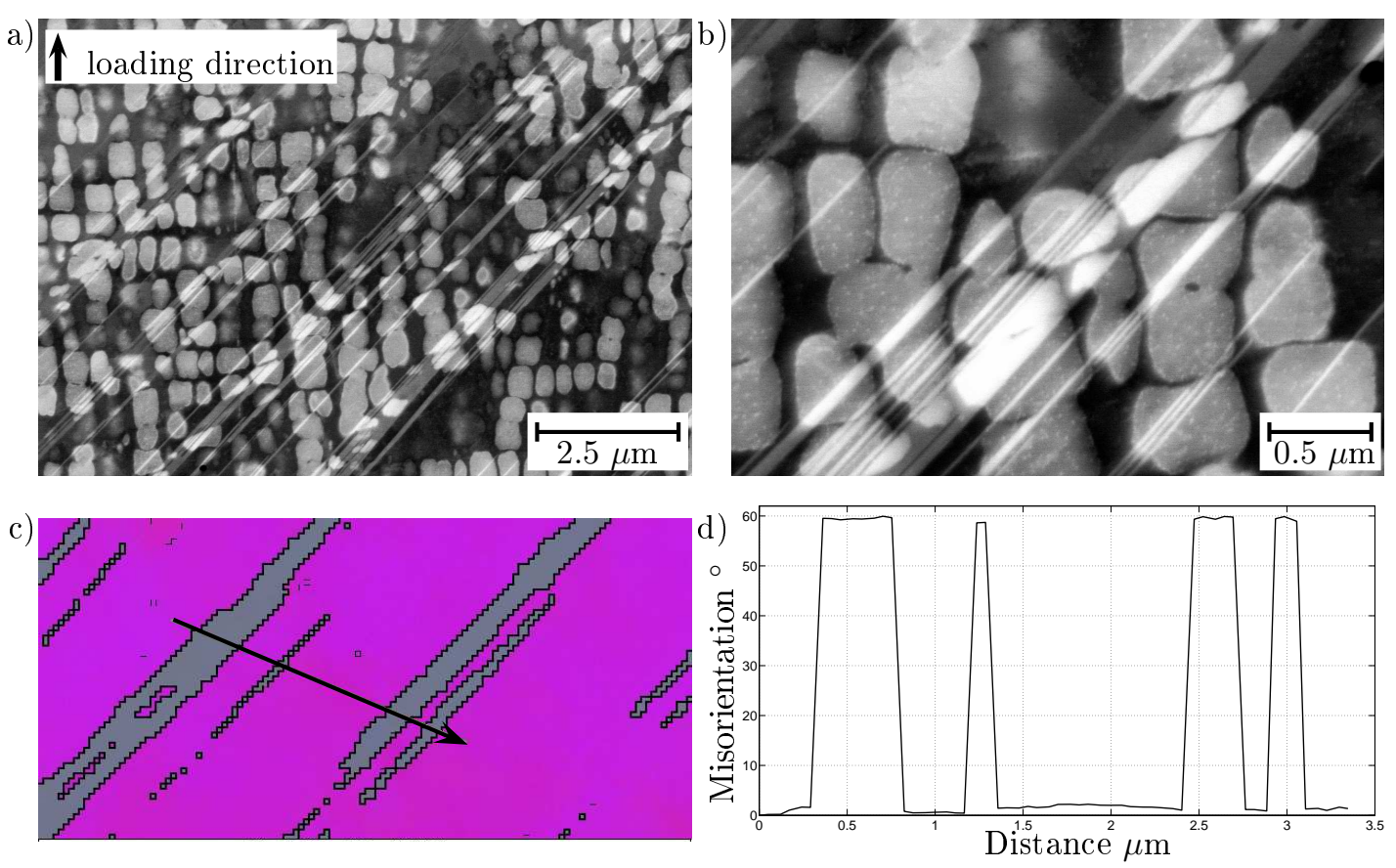

Figure 10: Deformation twinning a) in specimen B with b) magnification and confirmed in c) the EBSD map with the following d) the misorientation profile. 

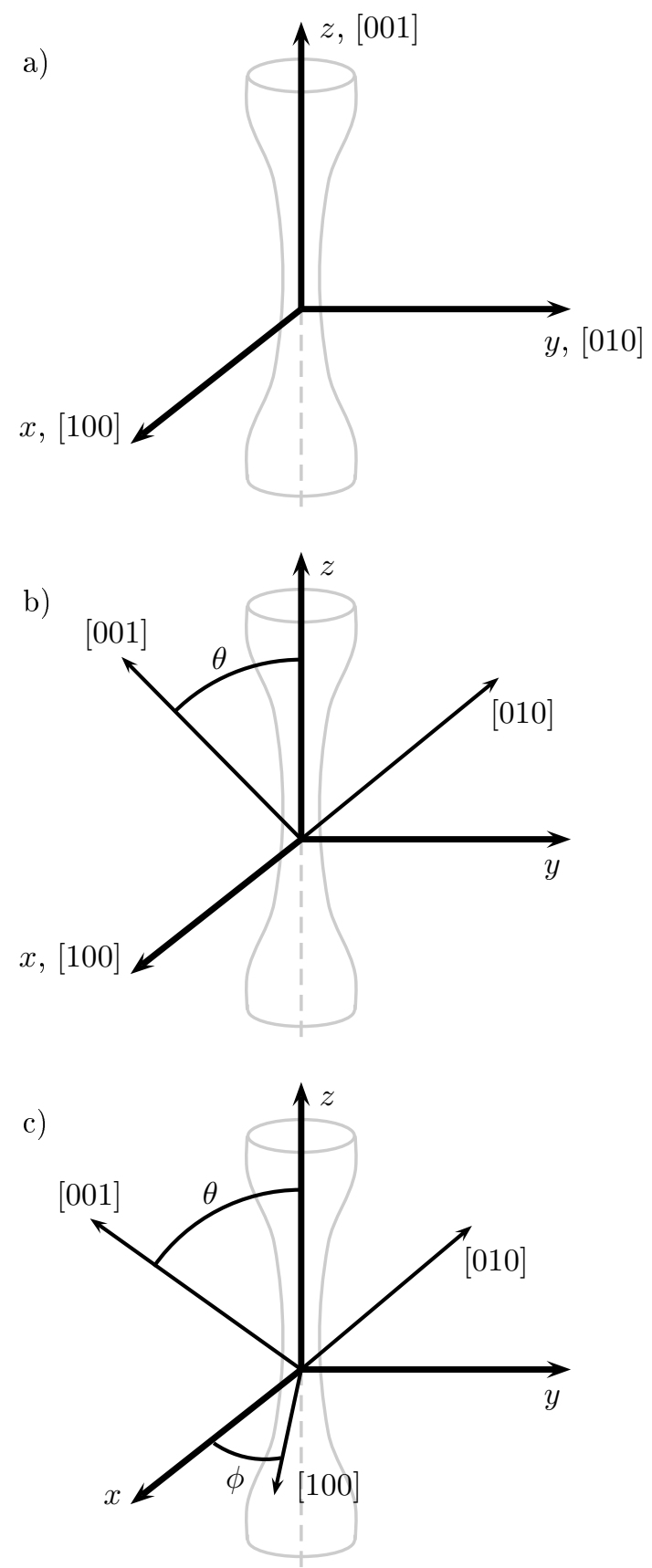

Figure 11: Definition of the specimen misalignments with respect to the nominal [001] crystal orientation. Loading in $z$-axis equal to a) [001], b) [011] and c) [111]. 


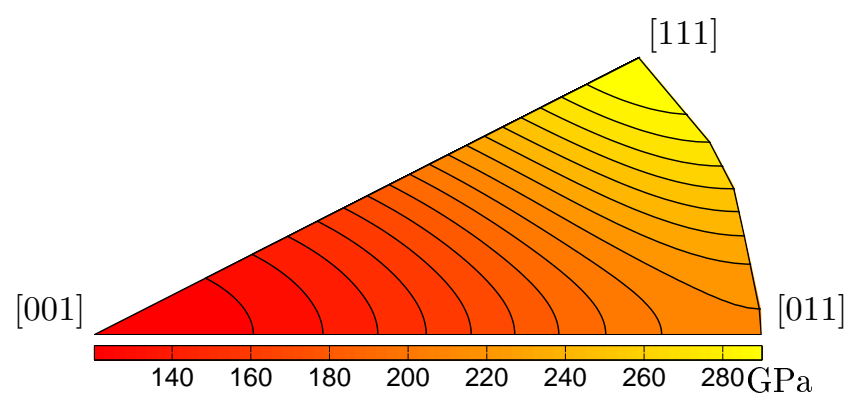

Figure 12: The numerical uniaxial moduli of elasticity for the respective crystal orientation in the stereographic triangle.

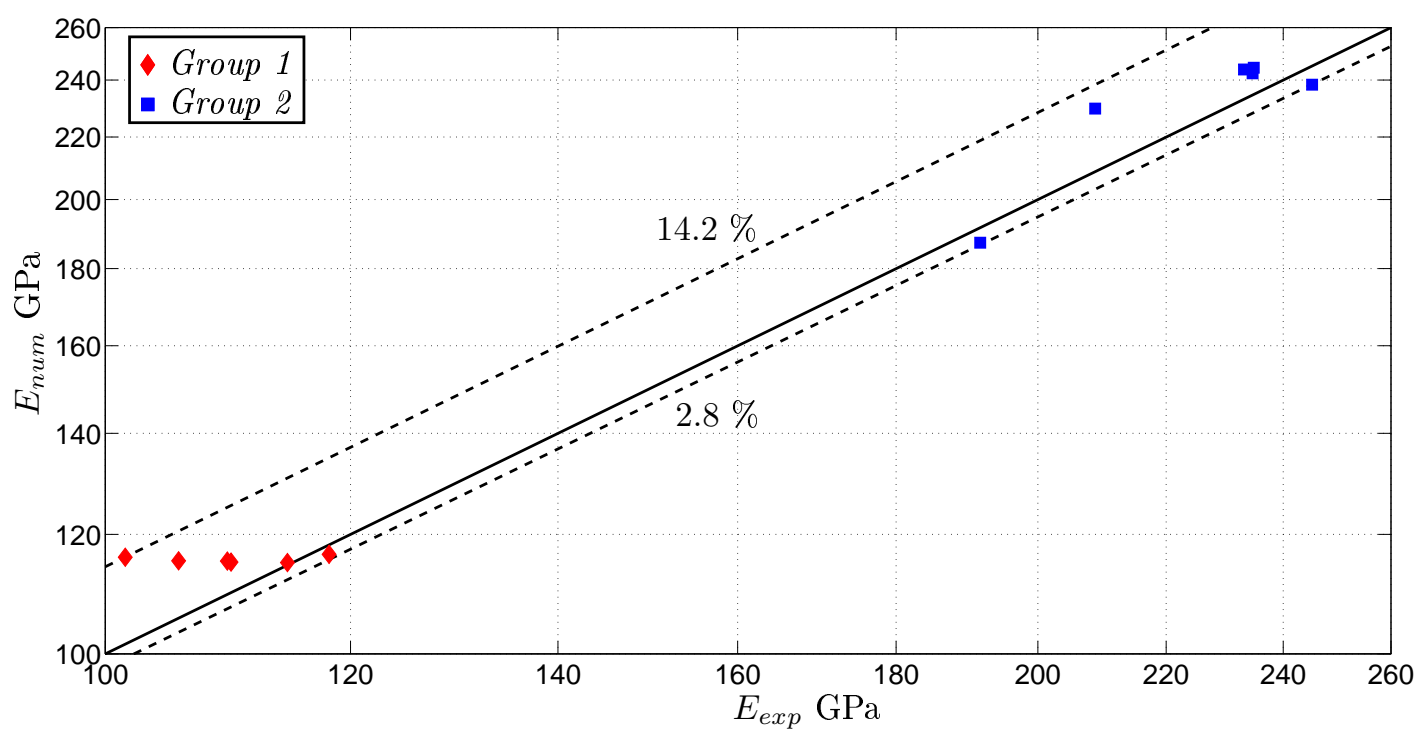

Figure 13: The comparison between the numerically obtained and experimentally determined modulus of elasticity. 


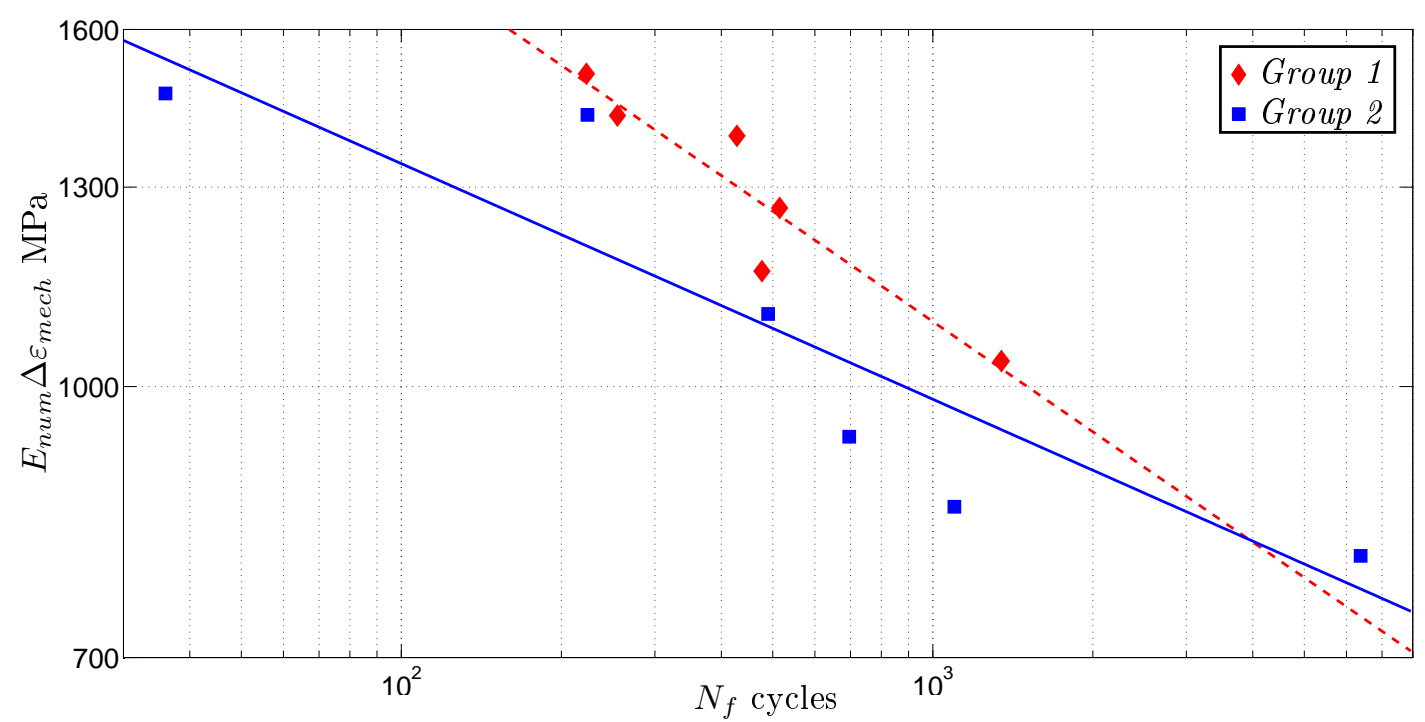

Figure 14: Mechanical strain range weighted with the respective numerical moduli of elasticity versus number of cycles to failure. 